12

\title{
Особенности ускорения тел миллиграммовой массы в рельсовых электромагнитных ускорителях с внешним импульсным магнитным полем
}

\author{
(С) С.А. Поняев, Б.И. Резников ", Р.О. Куракин, П.А. Попов, Б.Г. Жуков \\ Физико-технический институт им. А.Ф. Иоффре РАН, Санкт-Петербург, Россия \\ ๑ E-mail: boris.reznikov@mail.ioffe.ru
}

Поступило в Редакцию 4 октября 2018 г.

\begin{abstract}
В рельсотроне с внешним импульсным магнитным полем осуществлен разгон ударников из поликарбоната при атмосферном давлении в воздухе. Выполнен расчет динамики ускорения ударников. Проведено сравнение скорости в выходном сечении при питании цепей рельсотрона и внешнего поля от двух независимых источников энергии (схема 1) и при последовательном подключении цепей к одному источнику питания (схема 2). Установлено, что для двухмиллиметрового канала эффективный коэффициент эрозии рельсов, учитывающий только захваченную плазменным поршнем эрозионную массу, равен $1.5-1.7 \mathrm{mg} / \mathrm{C}$, a вовлеченная в движение эрозионная масса сравнима с массой ударника. Показано, что при использовании схемы 1 с опережающим подключением цепи внешнего поля ударник разгоняется при максимальной индукции поля, и скорость ударника в выходном сечении канала превышает $6 \mathrm{~km} / \mathrm{s}$.
\end{abstract}

DOI: 10.21883/PJTF.2019.02.47214.17550

Идея разгона твердых тел малого размера $(1-3 \mathrm{~mm})$ до высоких скоростей для доставки топлива в зону термоядерных реакций, диагностики горячей плазмы и управления режимами работы реактора остается актуальной с конца прошлого столетия [1-4]. Кроме того, инжекция пеллетов рассматривалась как средство для быстрого охлаждения плазмы и вывода тока в случае аварийной ситуации и опасности большого срыва $[3,5,6]$. Для установок типа ITER доставка вещества в центральные зоны реактора потребует скоростей пеллетов $5 \mathrm{~km} / \mathrm{s}$ и выше $[1,4-6]$. Другие направления использования быстрых макрочастиц включают моделирование активного космического эксперимента [7] и исследование высокоскоростного удара [8].

В работе [9] дано описание разработанного в ФТИ им. А.Ф. Иоффе компактного рельсового ускорителя, разгоняющего пластиковые кубики со стороной $1-2 \mathrm{~mm}$ и массой $1-10 \mathrm{mg}$ до скоростей 5-6 km/s. Потери энергии в каналах при катастрофической эрозии электродов на начальных участках разгона были преодолены наложением дополнительного внешнего импульсного магнитного поля, сонаправленного с собственным полем рельсотрона и сопоставимого с ним по величине $(\sim 10 \mathrm{~T})$. Цель настоящей работы - сравнение различных схем питания цепей рельсотрона и внешнего поля и верификация модели динамики ускорения.

Использовался малогабаритный ускоритель квадратного сечения $A=h^{2}$ калибром $h=2 \mathrm{~mm}$ с длиной канала $l=16 \mathrm{~cm}$, разгоняющий в воздухе при атмосферном давлении ударники массой $m_{0} \approx 10 \mathrm{mg}$. Погонная индуктивность рельсов $L^{\prime} \approx 0.21 \mu \mathrm{H} / \mathrm{m}$. Основные детали эксперимента и схема ускорителя описаны в [9]. Дополнительное внешнее магнитное поле в канале создавалось двумя последовательно соединенными одновит- ковыми катушками, расположенными симметрично по обе стороны канала ускорителя. Направление этого поля совпадало с направлением магнитного поля рельсотрона. В настоящей работе исследовались две схемы питания цепей рельсотрона и внешнего поля: питание каждой цепи от независимого источника энергии (схема 1) и подключение к единственному источнику энергии последовательного соединения рельсов-электродов и двух одновитковых катушек (схема 2). Схема 1 позволяла варьировать момент подачи импульса тока на витки подмагничивания и проводить начальную стадию разгона при значениях магнитной индукции, близких к максимальному. При использовании схемы 2 магнитная индукция внешнего поля $B \sim i_{w}(t)$ равна нулю в момент подачи импульса тока $i_{w}(t)$ на электроды и нарастает вместе с рабочим током, что значительно уменьшает скорость ударника на начальной стадии разгона. Измерение поля $B$ в канале проводилось датчиком, который предварительно калибровался с помощью специальных катушек Гельмгольца, рассчитанных на пропускание больших импульсных токов. Измерения показали, что поле мало менялось по длине канала, имело максимальное значение в середине канала и уменьшалось (не более $10 \%$ ) в казенной части и (в меньшей степени) к выходному сечению. Из-за диффузии поля коэффициент взаимной индукции витков уменьшался со временем. Скорость ударника измерялась в свободном полете по показаниям двух датчиков и относилась к измерительному сечению посередине между датчиками (см. пояснение к таблице). Скорость в выходном сечении $v(l)$ и время вылета $t(l)$ определялись с поправкой на торможение ударника в полете между выходным сечением канала и измерительным сечением. 
Результаты экспериментов

\begin{tabular}{|c|c|c|c|c|c|c|c|c|c|c|c|c|}
\hline \multirow{2}{*}{$\begin{array}{l}\text { Номер } \\
\text { опыта }\end{array}$} & \multirow{2}{*}{$B_{\max }, \mathrm{T}\left(N_{i p s}\right)$} & \multirow{2}{*}{$m_{0}, \mathrm{mg}$} & \multirow{2}{*}{$i_{w}^{\max }, \mathrm{kA}$} & $v_{f}$ & $v_{e s c}^{d}$ & $v_{e s c}^{t h}$ & $t_{e s c}^{d}$ & $t_{e s c}^{t h}$ & \multirow{2}{*}{$t_{d 1}, \mu \mathrm{s}$} & \multirow{2}{*}{$t_{d 2}, \mu \mathrm{s}$} & \multirow{2}{*}{$x_{d 1}, \mathrm{~mm}$} & \multirow{2}{*}{$x_{d 2}, \mathrm{~mm}$} \\
\hline & & & & \multicolumn{3}{|c|}{$\mathrm{km} / \mathrm{s}$} & \multicolumn{2}{|c|}{$\mu \mathrm{s}$} & & & & \\
\hline $6\left(t_{s}=27 \mu \mathrm{s}\right)$ & $8.9(2)$ & 10 & 94 & 5.73 & 6.31 & 6.30 & 50.3 & 50.2 & 106.5 & 124.3 & 340 & 442 \\
\hline 1 & 0 & 7.7 & 77.8 & 2.65 & 2.84 & 2.8 & 100 & 105 & 155.4 & 214 & 315 & 470 \\
\hline 2 & 0 & 7.9 & 120 & 4.06 & 4.36 & 4.32 & 78 & 78 & 113.6 & 152.8 & 313 & 472 \\
\hline 3 & $7.1(1)$ & 8.2 & 84.5 & 4.17 & 4.46 & 5.43 & 71 & 65 & 105.6 & 142.8 & 311 & 466 \\
\hline 4 & 0 & 7.9 & 86.7 & 2.87 & 3.08 & 3.14 & 96 & 100 & 148.8 & 203.2 & 318 & 474 \\
\hline 7 & $6(1)$ & 8 & 61 & 4.35 & 4.68 & 4.71 & 66 & 68 & 100.4 & 137.2 & 320 & 480 \\
\hline
\end{tabular}

Примечание. $N_{i p s}$ - число независимых источников питания цепей; $v_{e s c}^{t h}, t_{e s c}^{t h}, v_{e s c}^{d}, t_{e s c}^{d}-$ скорости и времена вылета ударника из выходного сечения, полученные из решения системы $(1)-(5)$ и рассчитанные по показаниям пленочных датчиков; $t_{d 1}, t_{d 2}-$ время пробоя ударником пленочных датчиков; $x_{d 1}, x_{d 2}$ - координаты датчиков, отсчитанные от торцевого сечения казенной части канала; $x_{f}=\left(x_{d 1}+x_{d 2}\right) / 2$ и $v_{f}=\left(x_{d 2}-x_{d 1}\right) /\left(t_{d 2}-t_{d 1}\right)-$ координата и скорость ударника в измерительном сечении.

Динамическая модель описывает штатный режим ускорения (без паразитных пробоев и утечек тока из плазменного поршня (ПП)). Суммарная сила, действующая на ударник, снижается силой давления ударносжатого газа $F_{d}$ и „эрозионной“ силой $F_{e r}$, возникающей при захвате ПП и вовлечении в движение вдоль оси канала части эрозионной массы, потерянной электродами.

При вычислении амперовой силы $F_{A}=F_{i}+F_{e}$ ПП рассматривается как непроницаемый тонкий токовый слой переменной массы. Здесь $F_{i}=L^{\prime} i_{w}^{2} / 2-$ сила, созданная рабочим током $i_{w}$, протекающим в рельсахэлектродах и в ПП, $F_{e}=B i_{w} h-$ сила, действующая на ток $i_{w}$, созданная внешним магнитным полем. Магнитная индукция $B=M^{\prime} i_{B} / h$ зависит от тока $i_{B}(t)$ в цепи катушек и от коэффициента взаимной индукции витков $M^{\prime}(t, x)$. При питании по схеме 2 $i_{B}=i_{w}, F_{e}=M^{\prime} i_{w}^{2}, F_{A}=\left(L^{\prime} / 2+M^{\prime}\right) i_{w}^{2}$. При питании по схеме $1 F_{e}=M^{\prime} i_{B} i_{w}, i_{w}(t) \neq i_{B}(t)$, что позволяет увеличить ток $i_{B}$ и силу $F_{e}$, подключив питание цепи внешнего поля в более ранний момент времени. В этом случае $F_{A}=L^{\prime} i_{w}^{2}(t) / 2+B\left(t+t_{s}\right) i_{w}(t) h$, где $t_{s}-$ сдвиг по времени момента включения цепи рельсотрона относительно момента включения питания цепи внешнего поля. При питании по схеме 2 время $t_{s}=0$.

Давление газа за ударной волной $(\mathrm{УB}) p_{2}$, степень сжатия $\rho_{2} / \rho_{1}(D)$ и связь между скоростями ударника $v$ и УВ $D$ находятся из решения системы уравнений сохранения на скачке [10]. Для УВ в воздухе при начальном давлении $p_{1}=1 \mathrm{bar}$ эти параметры интерполировались как функции $v$ по данным таблиц [11]. Эрозионная сила $F_{e r} \sim \dot{m}_{e r}\left(i_{w}\right)$ пропорциональна скорости изменения массы ПП $\dot{m}_{e r}$. Из-за малости коэффициента трения скольжения ударника о стенки канала $k_{f} \leq 0.065[12,13]$ сила трения скольжения ускоряемого тела не учитывалась.

Ускоряемый объект массой $m=m_{0}+m_{e r}+m_{s l}$ состоит из ударника массой $m_{0}$, эрозионной массы $m_{e r}$ захваченной ПП, и массы ударно-сжатого слоя $m_{s l}=\rho_{1} x_{s w} A$ протяженностью $\Delta=x_{s w}-x \quad\left(x, x_{s w}-\right.$ координаты ударника и УВ). Динамику ускорения объекта массой $m$ под действием амперовой силы $\left(F_{A}\right)$, силы давления газа, сжатого УВ $\left(F_{d}\right)$, и эрозионной силы $\left(F_{e r}\right)$ описывает система уравнений

$$
\begin{gathered}
\frac{d v}{d t}=\left(F_{A}+F_{d}+F_{e r}\right) / m, \\
F_{d}=-\left(p_{2}-p_{1}\right) A, \quad F_{e r}=-\dot{m}_{e r}\left(i_{w}\right) v, \\
\frac{d m_{e r}}{d t}=\dot{m}_{e r}\left(i_{w}\right), \\
m_{s l}=\rho_{1}(x+\Delta) A, \\
\frac{d \Delta}{d t}=\frac{v}{\rho_{2} / \rho_{1}-1} .
\end{gathered}
$$

При $v<c(c-$ скорость звука) задаются приближенные условия $m_{s l}=0, \Delta=0$.

Начальные условия имеют вид

$$
v(0)=0, \quad m(0)=m_{0}, \quad \Delta=0 .
$$

Поскольку зависимость $\dot{m}_{e r}\left(i_{w}\right)$ от калибра канала ранее не исследовалась, будем полагать, что количество захваченной эрозионной массы пропорционально перенесенному заряду. При $i_{w} \geq I_{0}$ используется выражение $\dot{m}_{e r}\left(i_{w}\right)=k_{e f f}\left(i_{w}-I_{0}\right)$, описывающее зависимости, полученные из экспериментов ([14], рис. 2,b), и содержащее два параметра: 1) $k_{e f f}=d m_{e r} / d i_{w}-$ эффективный коэффициент эрозии, учитывающий только эрозионную массу, вовлеченную в движение ПП; 2) $I_{0} \sim 10-20 \mathrm{kA}-$ пороговое значение тока, ниже которого экспериментальные данные по эрозии отсутствуют. В этой области влиянием эрозии на динамику ускорения можно пренебречь и считать $\dot{m}_{e r}=0$. Поскольку данные для двухмиллиметровых рельсотронов отсутствуют, значение $k_{e f f}$ оценивалось нами на основании согласования расчетных значений скоростей ударника в выходном сечении $v(l)=v_{e s c}^{t h}$ и скорости $v_{e s c}^{d}$, восстановленной по показаниям датчиков в опыте № 6, проведенном в работе [9] (см. таблицу). Расчеты показали, что приближенное равенство $v_{e s c}^{t h} \approx v_{e s c}^{d}$ достигается в диапазоне значений $k_{e f f}=1.5-1.7 \mathrm{mg} / \mathrm{C}$. Поэтому все последующие расчеты ускорения ударников в каналах 

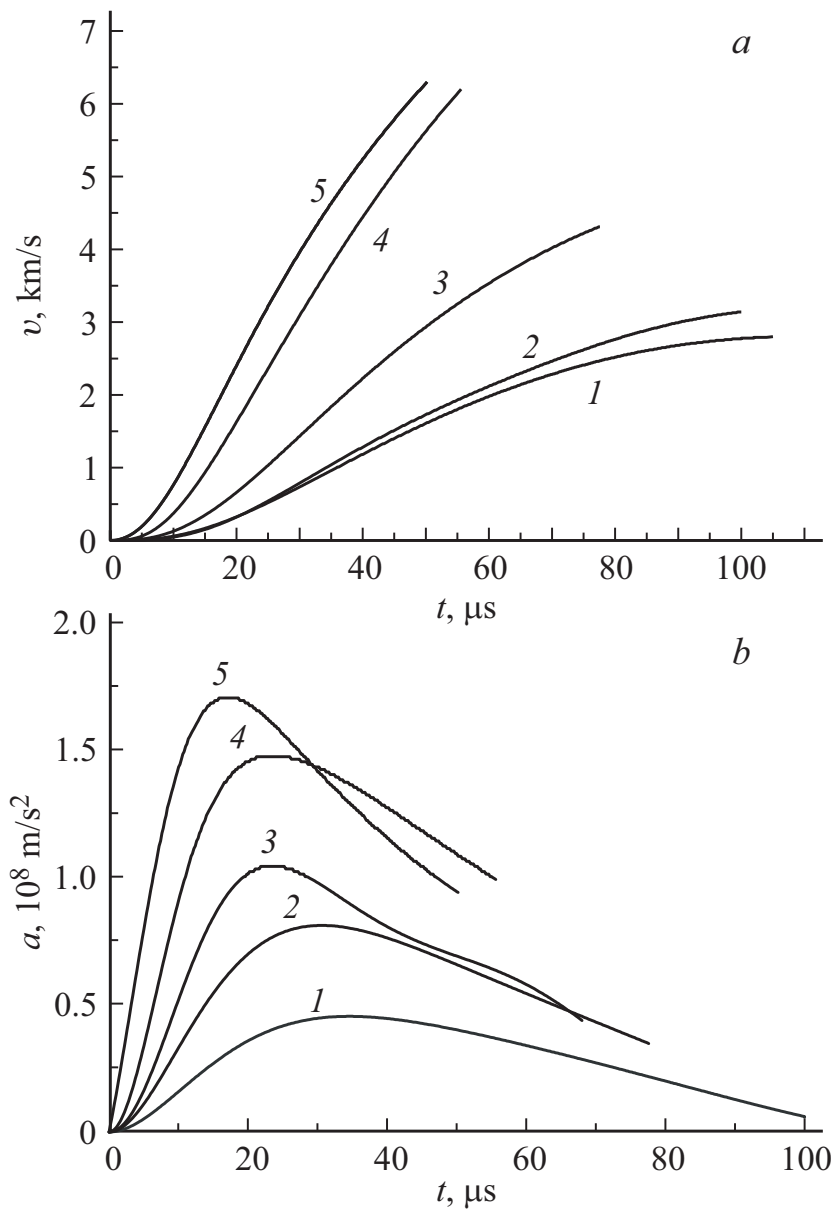

Рис. 1. Скорость ударника $(a)$ и ускорение $(b)$ для различных опытов: 1 - № 1,2 - № 2,3 - № 7,4 - № $6\left(t_{s}=0\right)$, 5 - № $6\left(t_{s}=27 \mu \mathrm{s}\right)$.

с медными рельсами калибром $2 \mathrm{~mm}$ проводились при значениях $k_{e f f}=1.6 \mathrm{mg} / \mathrm{C}, I_{0}=15 \mathrm{kA}$.

Результаты численных расчетов представлены в таблице и на рисунках. На рис. 1, $a$ показаны зависимости скорости ударника от времени, полученные при различных токах при отсутствии (кривые 1,2) и наличии внешнего поля (кривые 3-5). Из рис. 1, a видно, что время начального разгона ударника зависит от рабочего тока и внешнего поля и при меньших токах (кривые 1,2) достигает $\sim 15-20 \mu \mathrm{s}$. При наличии внешнего поля, создаваемого током цепи, подключенной к независимому источнику энергии, время разгона ударника значительно уменьшается, и скорость $v(l)$ при $t_{s}=27 \mu \mathrm{s}$ достигает величины $\sim 6.3 \mathrm{~km} / \mathrm{s}$.

Величина ускорения (рис. $1, b$ ) находится в диапазоне значений $10^{8} \mathrm{~m} / \mathrm{s}^{2}$ и является немонотонной функцией времени, что связано с немонотонностью ускоряющей и суммарной силы и увеличением ускоряемой массы. Другая особенность ускорения пеллетов связана с их малой массой. Для характерных токов 50-100 kA захваченная ПП эрозионная масса к моменту вылета ударника из канала становится сравнимой с массой ударника (рис. 2), что заметно снижает ускорение при приближении к выходному сечению. Масса ударно-сжатого слоя $m_{s l}=\rho_{1} x_{s w} A \sim h^{2}$ пренебрежимо мала по сравнению с массой ударника.

За исключением опыта № 3 (см. таблицу), отличие скоростей ударника в выходном сечении от значений, полученных по показаниям датчика, не превышает $2 \%$. Это подтверждает предположение, что величина $k_{e f f}$ в основном зависит от калибра канала. Превышение на $20 \%$ расчетной величиной скорости $v_{e s c}^{\text {th }}$ значения скорости $v_{e s c}^{d}$ в опыте № 3 можно объяснить, предположив, что при схеме 2 движение ударника на начальной стадии разгона было слишком медленным и сопровождалось потерями энергии на испарение жидкой фазы, а также разогрев и разрушение шунтирующих ПП токовых перемычек.

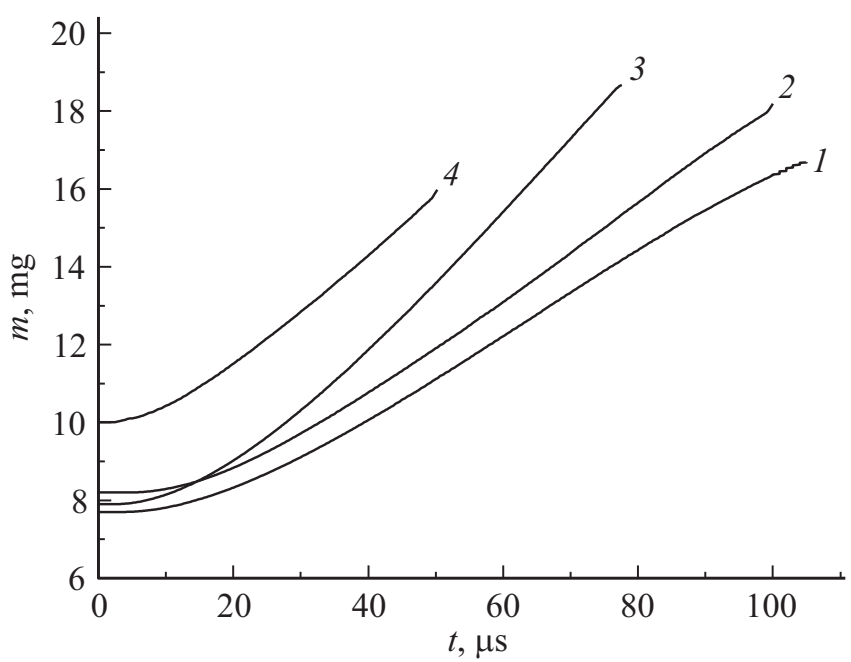

Рис. 2. Суммарная масса для различных опытов: 1 - № 1 , 2 - № 4, 3 - № 2, 4 - № $6\left(t_{s}=27 \mu\right.$ s).

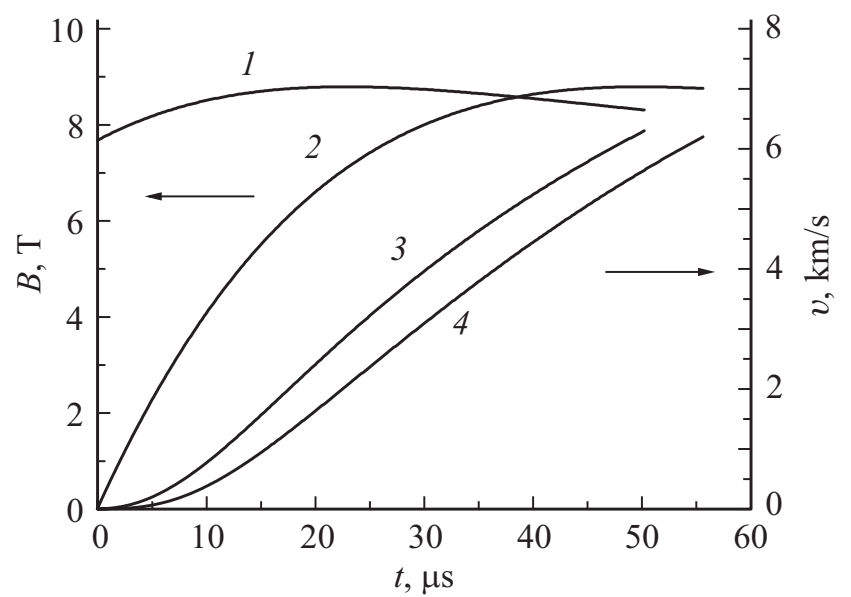

Рис. 3. Индукция внешнего магнитного поля $B(1,2)$ и скорость ударника $(3,4)$ при зависимости $B(t) .1,3$ - цепь питания рельсотрона включается на $27 \mu$ s позже цепи питания внешнего поля, 2,4 - одновременное включение питания цепей, $t_{s}=0$. 
Наибольший интерес представляет сравнительный анализ режимов ускорения при использовании двух схем подключения цепей питания внешнего поля и рельсотрона. На рис. 3 показаны зависимости от времени индукции внешнего поля и скорости ударника при подключении цепи внешнего поля на $27 \mu$ s раньше цепи рельсотрона (кривые 1,3 ) и одновременном включении цепей (кривые 2,4). Зависимости ускорения от времени в этих опытах представлены на рис. $1, b$ кривыми 5 и 4. В первом случае начальная стадия разгона происходит значительно быстрее (кривая 5 на рис. $1, b$ ), а большая ускоряющая сила обеспечивает разность скоростей ударника в $1 \mathrm{~km} / \mathrm{s}$ уже в начале разгона и уменьшение времени пребывания ударника в канале ускорителя. Таким образом, два источника энергии необходимы, когда требуется получить максимально возможные скорости. В противном случае можно предпочесть более простую схему питания с одним источником.

С.А. Поняев и П.А. Попов благодарят за финансовую поддержку исследований РФФИ (проект РФФИ 18-08-01503A).

\section{Список литературы}

[1] Milora S.L., Houlberg W.A., Lengyel L.L., Mertens V. // Nucl. Fusion. 1995. V. 18. P. 657-754.

[2] Combs S.K., Baylor L.R., Meitner S.J., Caughman J.B.O., Rasmussen D.A., Maruyama S. // Fusion Eng. Design. 2012. V. 87. P. 634-640.

[3] Kутеев Б.В. // ЖТФ. 1999. Т. 69. В. 9. С. 63-67.

[4] Combs S.K. // Rev. Sci. Instrum. 1993. V. 64. P. 1679-1698.

[5] Pégourié B. // Plasma Phys. Control. Fusion. 2007. V. 49. N 8. P. R87.

[6] Combs S.K., Love T.L, Jernigan T.S., Milora S.L. // Rev. Sci. Instrum. 1996. V. 67. P. 837-839.

[7] Poniaev S.A., Kurakin R.O., Reznikov B.I., Rozov S.I., Zhukov B.G., Chernyshov M.V. // Acta Astron. 2017. V. 135. P. 63-68.

[8] Poniaev S.A., Kurakin R.O., Sedov A.I., Bobashev S.V., Zhukov B.G., Nechunaev A.F. // Acta Astron. 2017. V. 135. P. 26-33.

[9] Жуков Б.Г., Куракин Р.О., Сахаров В.А., Бобашев С.В., Поняев С.А., Резников Б.И., Розов С.И. // Письма в ЖТФ. 2013. T. 39. B. 12. C. $63-70$.

[10] Зельдович Я.Б., Райзер Ю.П. Физика ударных волн и высокотемпературных гидродинамических явлений. М.: Наука, 1966. $686 \mathrm{c}$.

[11] Баум Ф.А., Орленко Л.П., Станюкович К.П., Челышев В.П., Шехтер Б.И. Физика взрыва / Под ред. К.П. Станюковича. М.: Наука, 1975. 704 с.

[12] Weeks D.A., Weldon W.F., Zowarka R.G. // IEEE Trans. Magn. 1989. V. 25. N 1. P. 580-586.

[13] Bowden F.P., Tabor D. The friction and lubrication of solids. Pt 2. Oxford: Oxford University Press, 1964. 544 p.

[14] Бобамев С.В., Резников Б.И., Жуков Б.Г., Куракин Р.О., Поняев С.А. // Письма в ЖТФ. 2015. Т. 41. В. 13. С. 64-71. 